\title{
Software
}

\section{Sotfware de prototipado para la arquitectura de la información: funcionalidad y evaluación}

\author{
Por Mario Pérez-Montoro y Lluís Codina
}

Resumen: El diseño y planificación de la arquitectura de la información de los distintos componentes de un sitio web pasa necesariamente por una propuesta de prototipado de la estructura arquitectónica de ese entorno informacional. El objetivo de este artículo es analizar y evaluar una serie de herramientas informáticas diseñadas para facilitar la creación de prototipos en el diseño y creación de sitios web.

Palabras clave: Prototipado de sitios web, Wireframes, Blueprints, Software, Prototipos, Maquetas, Aplicaciones en línea, Cloud computing.

Title: Prototyping software for information architecture: functionality and evaluation

Abstract: The planning and design of a web site information architecture necessarily involves a prototype proposal for the architectural structure of the informational environment. The aim of this paper is to analyze and evaluate a range of tools that facilitate the development of the wireframes in the design and creation of web sites.

Keywords: Web site prototyping, Software, Wireframes, Blueprints, Mockups, Online applications, Cloud computing.

Pérez-Montoro, Mario; Codina, Lluís. "Sotfware de prototipado para la arquitectura de la información: funcionalidad y evaluación". El profesional de la información, 2010, julioagosto, v. 19, n. 4, pp. 417-424.

DOI: 10.3145/epi.2010.jul.12

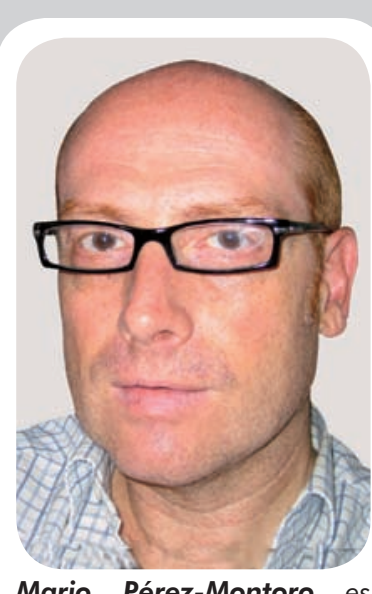

Mario Pérez-Montoro es doctor en filosofía y ciencias de la educación por la Univ. de Barcelona (UB), y master en organización de sistemas de documentación en la empresa por la UPC. Ha realizado estudios de postgrado en el Istituto di Discipline della Comunicazione de la Univ. di Bologna (Italia) y ha sido profesor visitante del CSLI (Center for the Study of Language and Information) de la Stanford Univ. (California, EUA). Investiga aspectos conceptuales, semánticos, epistemológicos y pragmáticos de la teoría de la información y la gestión del conocimiento. Es profesor de la Facultat de Biblioteconomia i Documentació de la UB.

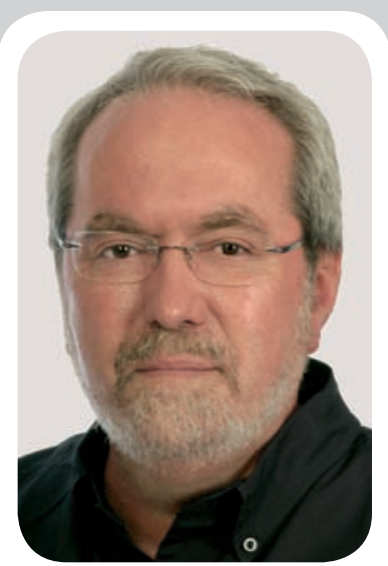

Lluís Codina es profesor titular del Departamento de Comunicación de la Universidad Pompeu Fabra. Imparte docencia en los estudios de periodismo y de comunicación audiovisual. Participa en máster oficiales y en programas de doctorado del Departamento de Comunicación. Coordina el grupo de investigación en Documentación Digital y Comunicación Interactiva, y forma parte del Grupo de Investigación en Periodismo, ambos reconocidos de la UPF -el último además por la Generalitat de Catalunya-. Su último libro (en coedición) se titula Web semántica y sistemas de información documental (Trea, 2009).

\section{Función del prototipado}

EL DISEÑO DE UN SITIO WEB suele incluir una propuesta de prototipado de su estructura arquitectónica (Pedraza-Jiménez, 2009), obteniéndose como resultado una serie de diagramas que cubren dos funciones esenciales:

a) Representar todos los aspectos básicos de los elementos arquitectónicos del sito web, componentes de cada uno de los sistemas (organización, etiquetado, navegación, búsqueda y vocabularios) que conforman la anatomía del sitio, la estructura de los contenidos y las relaciones existentes entre ellos.

b) Poder comunicar de forma eficaz esos aspectos a otros profesionales implicados en el proyecto (Brown, 2007; y Yusef et al., 2004).

Los dos principales tipos de diagramas o prototipos que se utilizan son los denominados blueprints $\mathrm{y}$ wireframes.
El blueprint (plano o mapa) es un esquema donde se representa la estructura arquitectónica del sitio web con todas sus páginas y donde se ponen de manifiesto las distintas relaciones existentes entre la página principal, las páginas que conforman el sitio y los contenidos que componen esas páginas.

Un wireframe (o maqueta), en cambio, es un prototipo arquitectónico de cada página del sitio web. Es un esquema que representa el contenido 
y la arquitectura de la información ( sin elementos gráficos) de cada una de las clases de página de un sitio.

\section{Herramientas de escritorio}

El primer grupo de soluciones analizadas en este artículo, y que se utilizan instaladas en el ordenador, son: Axure PR Pro 5.6, Visio profesional 2007, OmniGraffle 5, Denim 2.1, Conceptdraw Pro, Smartdraw 2010 y Pencil Project.

\subsection{Axure}

Junto a Visio, de Microsoft, Axure es uno de los programas de prototipado (wireframes y blueprints) más utilizados, para trabajar en Windows y Apple. Está en inglés $\mathrm{y}$ es de pago.

Ofrece una librería con una veintena de elementos gráficos $\mathrm{y}$ permite la incorporación de nuevos componentes gráficos externos.

Los diseños pueden exportarse a html, word, cvs, algunos formatos de imagen (bitmap, png, jpg o gif) y rp. En cambio, sólo importa directamente el propio formato rp de Axure. Permite anotaciones y notas a pie de página, la edición colaborativa con un sistema de control de

\section{Wireframes}

Son representaciones de las Fuente: http://isp.webopedia.com funciones, la estructura y el Las wireframes pueden hacerse contenido de una página o un sitio con papel y lápiz, con Power-Point, web. Separa los elementos gráficos Illustrator o Fireworks, pero mejor de los elementos funcionales, de forma que los diseñadores de la web pueden analizar fácilmente cómo interactuarán los usuarios con la web. Una wireframe típica incluye:

(1) elementos clave y su ubicación tales como cabecera, pie de página, navegación, objetos (tablas, fotos, vídeos, etc.), elementos de marca; (2) agrupación de elementos, tales como barras laterales, barras de navegación, áreas de contenidos, (3) etiquetado, título de la página, enlaces de navegación, cabeceras de objetos; y (4) marcadores de posición de contenidos, texto e imágenes

versiones y la creación de prototipos dinámicos y navegables.

En su página oficial aparece una importante biblioteca de plantillas de wireframes y blueprints, tutoriales de ayuda, un blog de novedades, un espacio de soporte sobre el producto, y un foro (en inglés) para los usuarios.

\section{http://www.axure.com}

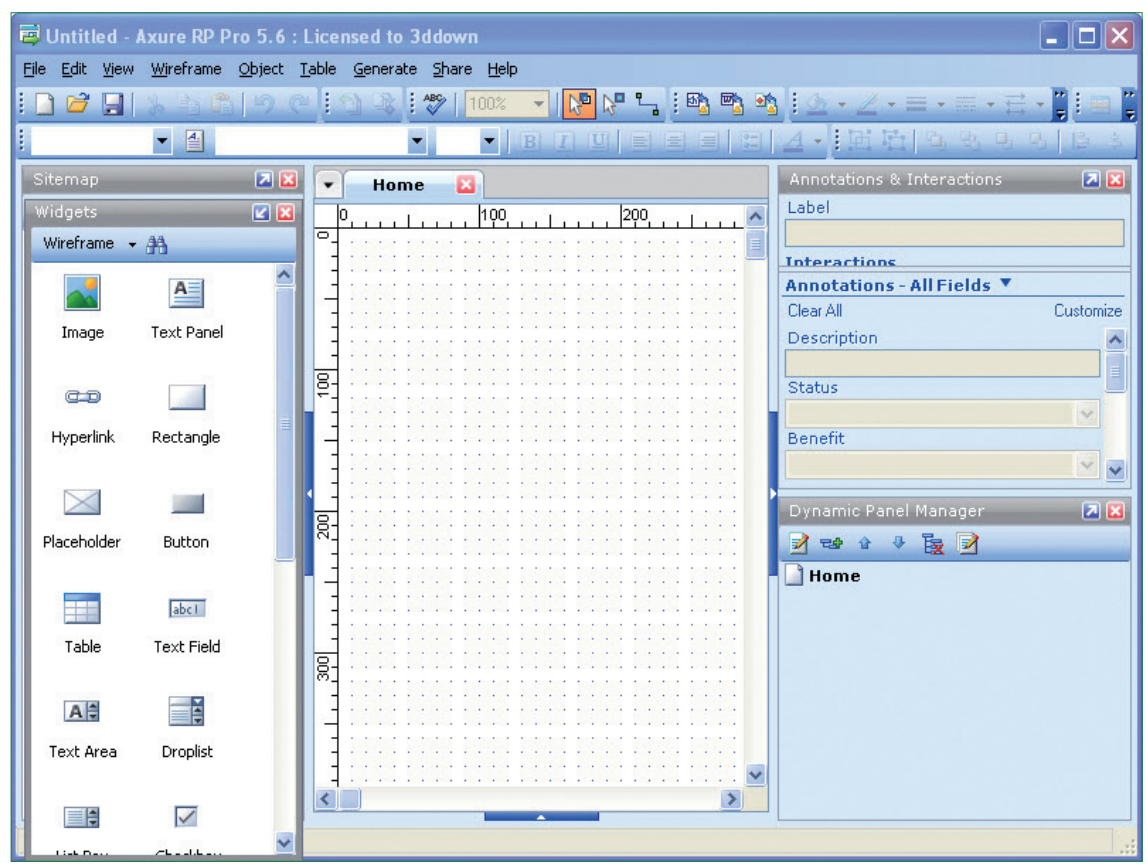

Figura 1. Vista parcial de los widgets (componentes) para wireframes de Axure usar los programas especializados presentados en este artículo.

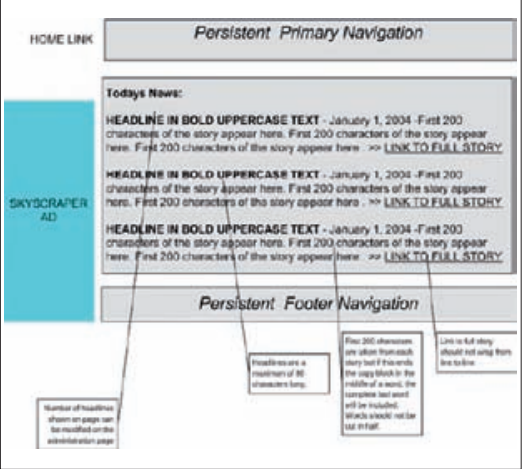

Ejemplo de wireframe

Fuente: http://articles.sitepoint.com/ article/process-and-documentation/5

\subsection{Visio profesional}

Es uno de los programas informáticos, exclusivamente para Windows, más versátiles y comúnmente utilizados en el desarrollo de propuestas de diseño web.

Se puede trabajar con una interfaz en inglés o en español. Ofrece una librería formada por una cincuentena de elementos gráficos, que puede ser enriquecida con elementos externos, y aporta una caja de búsqueda para facilitar el acceso al elemento a partir de su nombre.

Visio permite la exportación a pdf, html, sgv, tiff, jpeg, gif, png y visio; e importa html, sgv, tiff, jpeg, gif, png y visio. Soporta la inclusión de anotaciones y notas a pie de página, la edición colaborativa de prototipado y la creación de prototipos dinámicos.

En su página oficial aparece una importante biblioteca de plantillas de gráficas ( $\sin$ plantillas de wireframes y blueprints), tutoriales de ayuda, vídeos de formación, soporte y comentarios, y grupos de discusión (en español). 


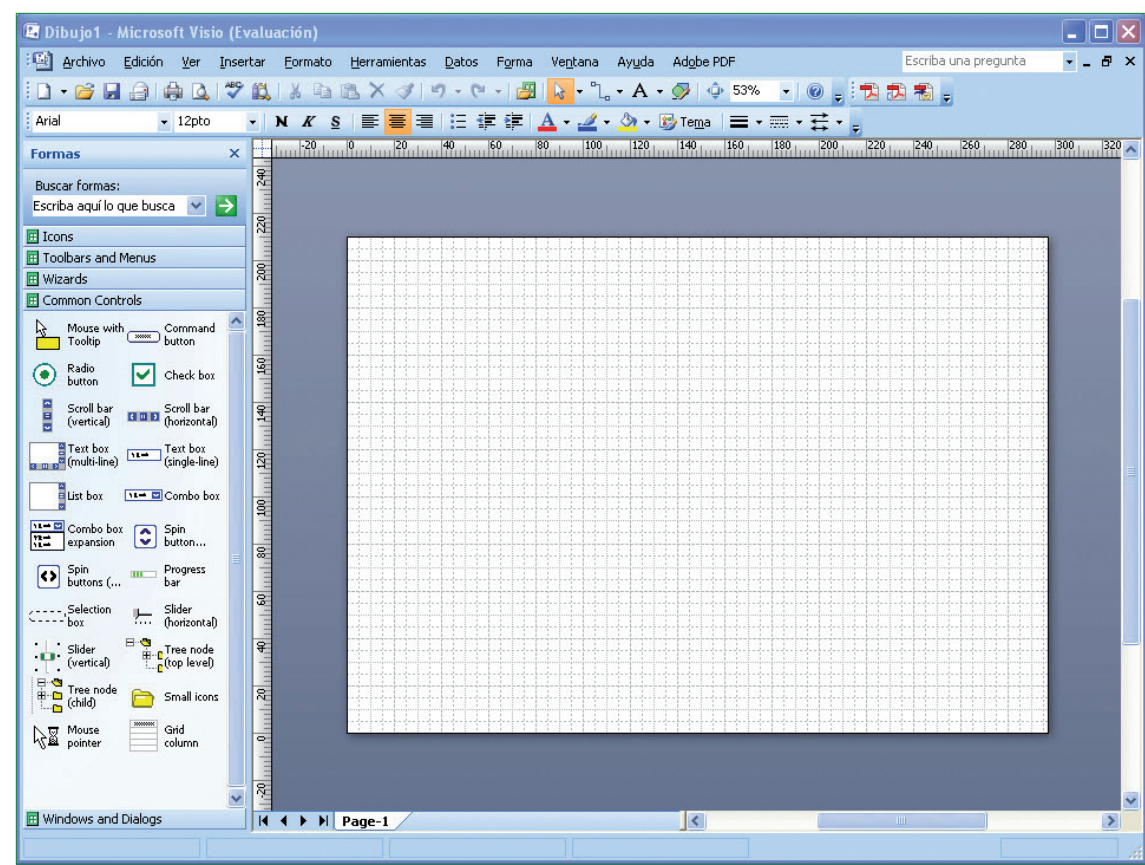

Figura 2. Interfaz de trabajo de Visio

http://office.microsoft.com/es-es/ visio/default.aspx

\subsection{OmniGraffle}

Es una de las herramientas de prototipado más utilizadas en el entorno de Apple. No existe versión para ningún otro sistema operativo, y está exclusivamente en inglés.

Es muy versátil para la creación gráfica en general, aunque permite también la creación de prototipado web.

No tiene librería de elementos gráficos, aunque permite la incorporación, gestión y edición de componentes gráficos externos. No es difícil obtener los elementos necesarios para el prototipado web (y de entornos móviles) como, por ejemplo, desde su propia página oficial.

Se exporta a omnigraffle, pdf, tiff, png, jpeg, eps, html, omnioutliner, svg, pict, photoshop, bmp y visio; y se importa de omnigraffle, visio, dot, xcode y eomodeler.

Como en los casos anteriores, ofrece la posibilidad de incluir anotaciones y notas a pie de página, prototipos dinámicos y, también, la edición colaborativa. En su página oficial podemos encontrar una
Admite exclusivamente la importación de su propio formato.

Permite la creación de prototipos dinámicos y navegables, pero no anotaciones y notas a pie de página, ni la edición colaborativa. Aunque su página oficial no incluye plantillas de wireframes ni blueprints, ofrece documentación de ayuda con ejercicios prácticos, un apartado de soporte y comentarios, y una lista de distribución donde solucionar dudas sobre su utilización.

http://dub.washington.edu:2007/ projects/denim

\subsection{Conceptdraw pro}

Aplicación comercial de escritorio en lengua inglesa y muy versátil para Windows y Apple.

Completada con el plug-in We$b$ Wave, ofrece una librería de más de 120 elementos gráficos, que puede ser enriquecida con otros externos. Incorpora el Site mapper wizard, que genera de forma automática el blueprint o mapa del sitio de una web ya existente.

Permite la exportación a pdf, html, xml, power point, macromedia flash, los principales formatos gráficos, svg, y conceptdraw; y la importación de xml, los principales formatos gráficos, power point, bmp, visio y conceptdraw. Aunque no incluye la opción de insertar anotaciones, sí permite las notas a pie de página, la edición colaborativa y la creación de prototipos dinámicos.

En su página oficial muestra una importante biblioteca de plantillas de gráficas, tutoriales de ayuda, un espacio de soporte y un espacio (community) en inglés para sus usuarios.

\section{http://www.conceptdraw.com}

\subsection{Smartdraw}

Es una versátil herramienta de escritorio, en inglés y sólo para Windows, diseñada para la realización de tareas gráficas en el contex- 


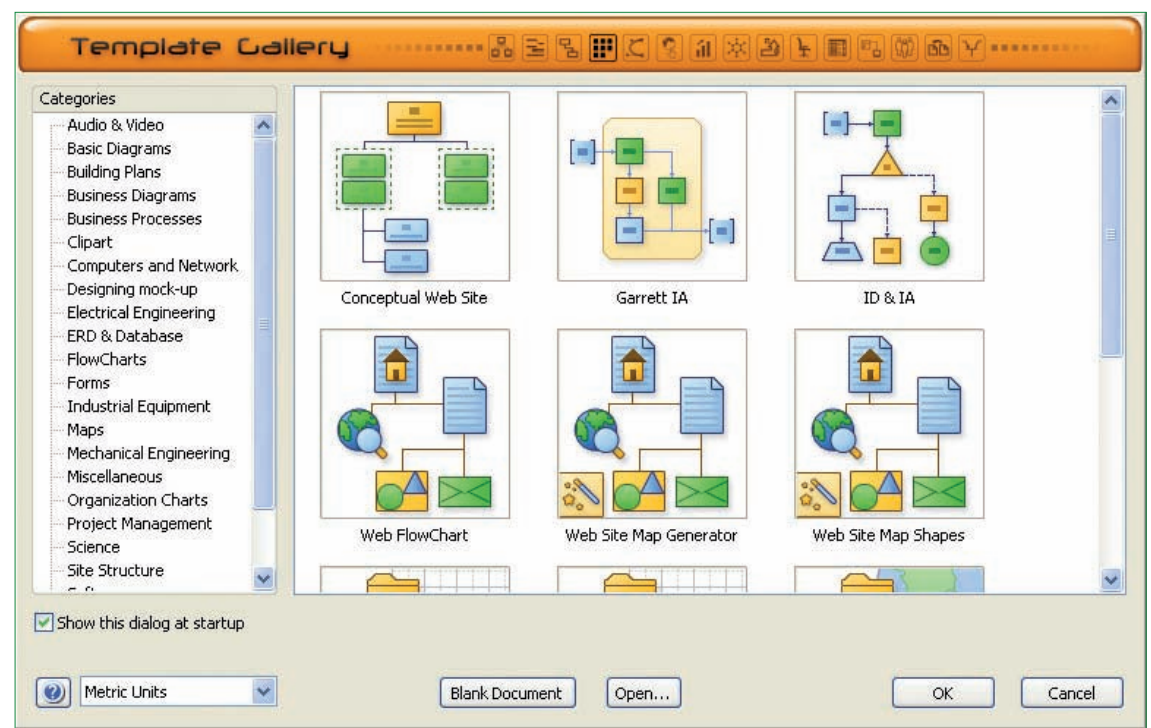

Figura 3. Galería de herramientas de diseño, y tipos de diagramas que pueden realizarse con Conceptdraw pro

to de las organizaciones, que incluye también recursos para el prototipado de webs.

Proporciona una librería con un extenso conjunto de elementos gráficos. Permite la gestión y edición de los elementos que ofrece y la incorporación de nuevos componentes gráficos externos.

Facilita la exportación a diferentes formatos como pdf, word, power point, exel, word perfect, formatos gráficos, html, metalife, y smartdraw; pero sólo importa los formatos visio y smartdraw. Permite anotaciones y notas a pie de página, la edición colaborativa de prototipado y la creación de prototipos dinámicos e interactivos.

En su página oficial aparece, entre otros recursos, una importante biblioteca de plantillas de wireframes y blueprints, tutoriales de ayuda, un glosario para la aclaración de términos y un foro para sus usuarios.

\section{http://www.smartdraw.com}

\subsection{Pencil project}

Programa gratuito, de código abierto, en inglés, para Windows y GNU/Linux. También puede ser instalado como una extensión (addon) en el navegador Firefox.

Es una solución muy flexible que ofrece una librería formada por más de 50 elementos gráficos, que admiten una edición posterior y la incorporación de nuevos elementos gráficos externos. Provee la exportación a html, png, openoffice, word, pdf y pensil; pero sólo importa su propio formato.

Soporta la creación de prototipos dinámicos y anotaciones; pero no las notas a pie de página ni la edición de proyectos de creación colaborativa de prototipado. Su página oficial ofrece ejemplos y plantillas de wireframes y blueprints, tutoriales de ayuda y un espacio para la discusión.

\section{http://www.evolus.vn/pensil}

\section{Herramientas en línea}

En los últimos años ha aparecido una amplia gama de aplicaciones basadas en el llamado cloud computing, es decir, que se ejecutan en una web a través de un navegador, sin necesidad de instalar nuevo software en nuestro ordenador.

Los datos y documentos creados quedan guardados en servidores remotos de la internet, por lo cual se puede acceder a ellos desde cualquier ordenador del planeta. Por esta misma razón facilitan la edición colaborativa dentro de grupos de trabajo dispersos. Natu- ralmente, todas estas son funciones con las que las aplicaciones de escritorio, por definición, no pueden competir. Además, al ejecutarse desde cualquier navegador estándar, son independientes del sistema operativo del usuario (Windows, $\mathrm{Li}$ nux, Mac, etc.).

En general, las soluciones en línea son menos sofisticadas que las de escritorio, pero en contrapartida son más baratas y ofrecen versiones a coste cero. Esto permite que puedan ser utilizadas también en entornos como el de la enseñanza.

Vamos a analizar las siguientes: Mockflow, iPlotz, Pidoco, Lovely Chart, MockingBird y Lumzy (las versiones evaluadas son las que estaban disponibles en mayo de 2010).

\subsection{Mockflow}

Es uno de los productos más completos de esta comparativa, con una interfaz muy elegante y de uso sumamente intuitivo, que proporciona un gran número de elementos (o widgets) de diseño, tanto de tipo estándar como para móviles.

Ofrece casi todo lo que se puede esperar de un programa de prototipado: control de versiones, colaboración en tiempo real, anotaciones, diseño basado en esquemas, importación de esquemas predefinidos o generación de mapas del web, etc. Adicionalmente, dispone de una aplicación gemela de escritorio para trabajar en modo offline. Por último, es uno de los programas que disponen de versión gratuita (tipo basic) sin ninguna restricción funcional, únicamente en relación al número de proyectos y de páginas de cada proyecto. Ideal, por tanto, para quienes buscan buenas herramientas para llevar a las aulas.

\section{http://www.mockflow.com}

\section{2. iPlotz}

Es otro de los pesos pesados de las aplicaciones online. Comparte muchas de las características de su gran rival comercial en esta 


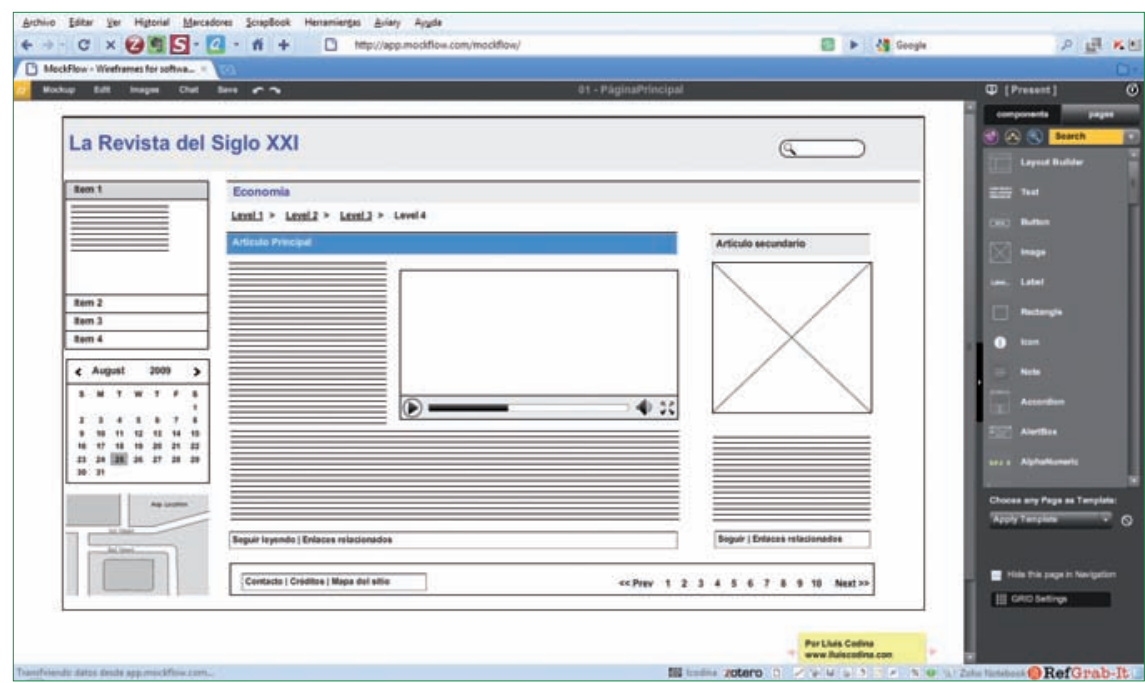

Figura 4: Interfaz de una aplicación de prototipado en línea con un ejemplo de wireframe en la ventana de edición (Mockflow)

área (Mockflow), como su elevada especialización, el gran número de elementos (sobre un centenar), la aportación de elementos móviles, y wireframes dinámicos, entre otras. portación, especialmente en html, con un resultado de una fidelidad extraordinaria. $\mathrm{Su}$ interfaz es eficaz y muy eficiente, pero quizá un poco intimidatoria comparada con la sencillez de Mockflow. Parte de esa complejidad procede del mayor número de funciones que presenta iPlotz frente a sus competidoras. Es la aplicación en línea que proporciona más opciones de exportación, así como funciones específicas de gestión de proyectos y de edición colaborativa. Ofrece, además, una versión gratuita sin limitaciones
Destaca por sus opciones de ex-

funcionales (con limitación del número de proyectos).

http://www.iplotz.com

\subsection{Pidoco}

Pidoco completa el trío de las aplicaciones en línea que en aportación de funciones puede compararse sin complejos con sus competidoras de escritorio.

Algunos de sus puntos fuertes son la eficacia de sus exportaciones a Word y html, la posibilidad de dos vistas diferentes del prototipado una del tipo mano alzada y otra más clásica (ortogonal)-, sus opciones de trabajo colaborativo, y el poder llevar a cabo tests de usabilidad en modo remoto.

\section{http://www.pidoco.com}

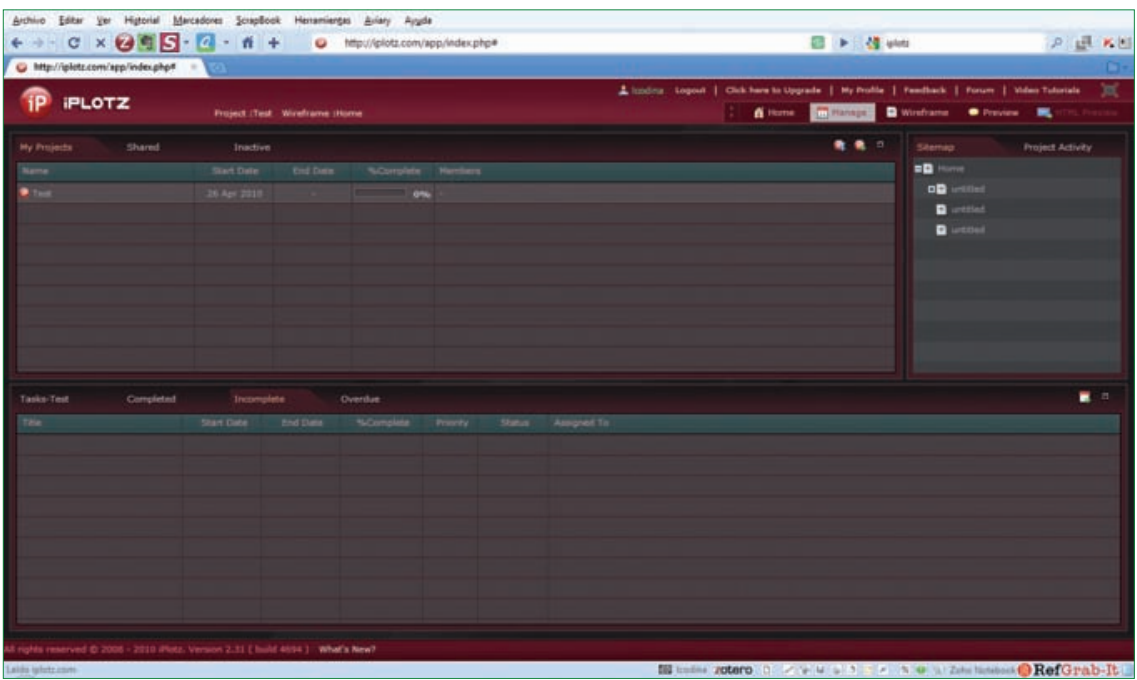

Figura 5: Gestor de proyectos de una aplicación en línea (iPlotz)

\subsection{Lovely chart}

Es la única de las aplicaciones en línea consideradas aquí con un grado de versatilidad comparable a las de escritorio, pero la paga en una baja especialización en el campo que nos ocupa, ofreciendo pocos elementos para prototipado. Es decir, se trata del más flexible de los programas en línea, pero el menos especializado.

Destaca la calidad de su interfaz, con soluciones innovadoras, y la facilidad de edición de los elementos una vez puestos en el lienzo: dimensiones, color, establecimientos de conexiones, etc. Forma parte del grupo de honor de las aplicaciones online con versión gratuita. De todos modos, su versión de pago es realmente barata $(29 €)$.

\section{http://www.lovelycharts.com/}

\subsection{MockingBird y Lumzy}

Por último, incluimos en un mismo apartado dos soluciones que comparten varios rasgos. En primer lugar, ambas ofrecen únicamente versiones gratuitas (en el caso de MockingBird, al menos mientras se mantenga en versión beta). Lumzy indica que ofrecerán soporte al usuario, pero parecen apuntar, sin explicitarlo, que vendrá con algún tipo de licencia de pago.

En segundo lugar, sobre todo comparadas con las tres primeras, son las dos soluciones menos especializadas, aunque ambas son muy notables en cuanto al número de elementos que proporcionan y, sobre todo, en su extremada facilidad de uso.

\section{Evaluación de las herramientas}

Como es sabido, todo modelo de evaluación incorpora un cierto grado inevitable de subjetividad. $\mathrm{Ni}$ tan solo los sistemas de evaluación que siguen los grandes organismos internacionales, como Naciones Unidas o la $O C D E$, en sus clasifica- 


\begin{tabular}{|l|c|c|c|c|c|}
\hline Herramienta & $\begin{array}{c}\text { Operativi- } \\
\text { dad }\end{array}$ & $\begin{array}{c}\text { Funciona- } \\
\text { lidad }\end{array}$ & Plantillas & Soporte & Total \\
\hline iPlotz & 5 & 11 & 0 & 2 & 18 \\
\hline Smartdraw & 0 & 14 & 2 & 2 & 18 \\
\hline Conceptdraw & 0 & 14 & 2 & 2 & 18 \\
\hline Pencil project & 3 & 11 & 2 & 2 & 18 \\
\hline OmniGraffle & 0 & 13 & 2 & 2 & 17 \\
\hline Axure & 1 & 12 & 2 & 2 & 17 \\
\hline MockFlow & 3 & 11 & 1 & 2 & 17 \\
\hline Visio & 1 & 13 & 0 & 2 & 16 \\
\hline Pidoco & 3 & 7 & 0 & 2 & 12 \\
\hline Lovely chart & 3 & 7 & 0 & 2 & 12 \\
\hline Denim & 3 & 6 & 0 & 2 & 11 \\
\hline MockingBird & 3 & 5 & 0 & 1 & 9 \\
\hline Lumzy & 3 & 5 & 0 & 1 & 9 \\
\hline
\end{tabular}

Tabla 1. Ranking de todas las herramientas

ciones están libres de un cierto grado de discrecionalidad. No pretendemos por tanto presentar un modelo de análisis perfecto y objetivo al cien por cien, absolutamente indiscutible, sino una orientación que resulte, al menos conveniente (ayuda a conocer mejor los programas y a tomar decisiones al situarlos en un marco comparativo) y racional (nos hemos centrado en los aspectos principales de cada programa como plataforma y en sus funcionalidades de prototipado web).

Por ello vamos a analizar los siguientes parámetros: operatividad, funcionalidad, plantillas y soporte.

\section{Operatividad}

Para calcular el grado de operatividad de la herramienta utilizamos tres indicadores:

- sistemas operativos en los que corre (le otorgamos 0 puntos si sólo corre en un sistema, 1 punto en dos, y 2 puntos si funciona en tres sistemas operativos o es en línea);

- distintas lenguas en la que se ofrece su interfaz (le asignamos 0 puntos si su interfaz se ofrece en una sola lengua, 1 punto en dos, y 2 puntos si se ofrece en más de dos lenguas); $\mathrm{y}$

- tipo de licencia de la herramienta (0 puntos si es de pago y 1 punto si ofrece, independientemente de la versión para la evaluación, una versión gratuita).

\section{Funcionalidad}

Para evaluarla hemos utilizados los siguientes indicadores y sistema de puntuación:

- grado de versatilidad (0 puntos si permite sólo el prototipado, 1 punto si permite además otro tipo de representación gráfica, y 2 puntos si incluye además dos o más tipos de representación gráfica);

\begin{tabular}{|l|c|c|c|c|c|}
\hline Herramienta & $\begin{array}{c}\text { Operativi- } \\
\text { dad }\end{array}$ & $\begin{array}{c}\text { Funciona- } \\
\text { lidad }\end{array}$ & Plantillas & Soporte & Total \\
\hline Smartdraw & 0 & 14 & 2 & 2 & 18 \\
\hline Conceptdraw & 0 & 14 & 2 & 2 & 18 \\
\hline Pencil project & 3 & 11 & 2 & 2 & 18 \\
\hline OmniGraffle & 0 & 13 & 2 & 2 & 17 \\
\hline Axure & 1 & 12 & 2 & 2 & 17 \\
\hline Visio & 1 & 13 & 0 & 2 & 16 \\
\hline Denim & 3 & 6 & 0 & 2 & 11 \\
\hline
\end{tabular}

Tabla 2. Ranking de las herramientas de escritorio
- elementos estándar de prototipado que incluye ( 0 puntos si ofrece menos de 20 elementos; 1 punto si ofrece más de 20 y 2 puntos si permite la incorporación de elementos externos);

- elementos de prototipado para interfaces móviles $(0$ puntos si no los incorpora y no permite su incorporación; 1 punto si no los incorpora pero permite su importarlos y 2 puntos si los incorpora directamente);

- tipos de formatos a los que permiten exportar (0 puntos si sólo permite la exportación en su formato; 1 punto si permite la exportación a tres o menos formatos y 2 puntos si permite la exportación a más de tres formatos);

- tipos de formatos que permiten importar (0 puntos si sólo permite la importación desde su formato; 1 punto si permite la importación desde tres o menos formatos y 2 puntos si permite la importación desde más de tres formatos);

- creación y edición colaborativa ( 0 puntos si no la permite y 1 punto si la permite), la anotación (0 puntos si no la permite y 1 punto si la permite), las notas a pie de página (0 puntos si no las permite crear y 1 punto si permite su inserción);

- prototipos dinámicos (0 puntos si no los permite crear y 1 punto permite su inserción);

- wireframes (0 puntos si no los permite crear y 1 punto permite su implementación); y

- blueprints (0 puntos si no los permite crear y 1 punto permite su implementación).

\section{Plantillas}

Respecto a las plantillas hemos utilizados como indicadores:

- inclusión de plantillas de wireframes ( 0 puntos si no las ofrece y 1 punto si las ofrece); e

- inclusión de plantillas de blueprints ( 0 puntos si no las ofrece y 1 punto si las ofrece). 


\begin{tabular}{|l|c|c|c|c|c|}
\hline Herramienta & $\begin{array}{c}\text { Operativi- } \\
\text { dad }\end{array}$ & $\begin{array}{c}\text { Funciona- } \\
\text { lidad }\end{array}$ & Plantillas & Soporte & Total \\
\hline iPlotz & 5 & 11 & 0 & 2 & 18 \\
\hline MockFlow & 3 & 11 & 1 & 2 & 17 \\
\hline Pidoco & 3 & 7 & 0 & 2 & 12 \\
\hline Lovely chart & 3 & 7 & 0 & 2 & 12 \\
\hline MockingBird & 3 & 5 & 0 & 1 & 9 \\
\hline Lumzy & 3 & 5 & 0 & 1 & 9 \\
\hline
\end{tabular}

Tabla 3. Ranking de las herramientas online

\section{Soporte}

Por último, la variable relacionada con el soporte o grado de asistencia ha sido evaluada a partir de:

- tutoriales (0 puntos si no los incluye y 1 punto si los incluye); y

- blogs o espacios de ayuda (0 puntos si no los ofrece y 1 punto si los ofrece).

Los resultados globales de la evaluación de la totalidad de las herramientas analizadas se recogen, en forma de ranking, en la tabla 1. Los resultados segregados por tipos de solución se incluyen, también en forma de ranking, en las tablas 2 (herramientas de escritorio) y 3 (herramientas en línea).

\section{Conclusiones}

\section{Resultados globales}

En primer lugar, como vemos en la tabla 1, el ranking está dominado por cuatro herramientas que obtienen una valoración de 18 puntos: iPlotz, Smartdraw, Conceptdraw y Pencil project. Aunque iPlotz, presenta una funcionalidad media-alta (11 puntos), se sitúa muy bien en el ranking gracias al índice de operatividad más alto (5 puntos) de las herramientas analizadas. Smartdraw y Conceptdraw presentan los mismos valores, destacando el mayor nivel de funcionalidad de las herramientas evaluadas (14), pero penalizadas por su nula operatividad (0 puntos). Pencil project se coloca en este primer grupo gracias a una operatividad alta (3 puntos), una funcionalidad media-alta (11 puntos) y una puntuación máxima respecto a los indicadores plantillas y soporte (2 puntos cada una).

Seguidos muy de cerca, en la rece un grupo de herramientas formado por las soluciones OmniGraffle, Axure y MockFlow, con una valoración de 17 puntos cada una. OmniGraffle destaca por su alta funcionalidad (14 puntos, la segunda más alta) aunque se ve penalizado por su baja operatividad (0 puntos). Axure ve equilibrada su menor funcionalidad (13 puntos), con una operatividad más alta (1 punto). MockFlow justifica la puntuación obtenida a partir de una funcionalidad media-alta (11 puntos) pero con una operatividad alta (3 puntos, el segundo valor más alto).

Muy cercana a este segundo grupo aparece Visio, con una valoración de 16 puntos obtenida a partir de una funcionalidad muy alta (13 puntos, el segundo valor más alto) pero con una operatividad más escasa (1 punto) y sin puntuar en el parámetro plantillas.

En la zona media-baja del ranking, aparecen las herramientas Pidoco, Lovely chart y Denim. Pidoco y Lovely chart obtienen la misma puntuación total (12 puntos) y los mismos valores respecto a los parámetros operatividad (3), funcionalidad (7), plantillas (0) y soporte (2). Denim, aunque presenta una operatividad alta (3 puntos), obtiene sólo 11 puntos a partir de una funcionalidad escasa (6 puntos, la segunda más baja) y no puntuar en el parámetro relacionado con las plantillas. segunda posición del ranking, apa-
El ranking se completa con MockingBird y Lumzy, con 9 puntos cada una. Aunque presenten una operatividad alta (3 puntos, el segundo valor más alto), la baja puntuación total se justifica por obtener el valor más bajo de funcionalidad (sólo 5 puntos) de las herramientas evaluadas, por obtener sólo un punto con respecto al soporte y por no puntuar en el parámetro de las plantillas.

\section{Herramientas de escritorio}

Las que presentan la mejor evaluación son Smartdraw, Conceptdraw y Pencil project, con 18 puntos cada una. Smartdraw y Conceptdraw obtienen sus altas puntaciones a partir de los parámetros funcionalidad (14, la más alta de todas las herramientas evaluadas), plantillas (2) y soporte (2); pero presentando una nula operatividad (0). Pencil project, en cambio, obtiene esa puntuación a partir de la operatividad (3, la más alta de las herramientas de escritorio), plantillas (2) y soporte (2); pero presentando la segunda puntuación más baja respecto a su funcionalidad (11 puntos).

El siguiente grupo de herramientas de escritorio en el ranking está formado por OmniGraffle y Axure con 17 puntos cada una. OmniGraffle basa principalmente la puntuación obtenida en los parámetros funcionalidad (13), plantillas (2) y soporte (2), pero presentando también una nula operatividad (0). Axure la obtiene también a partir de los mismos valores pero con una menor funcionalidad (12). Visio, en cambio, presenta una funcionalidad un poco más alta (13) pero falla en el tema de las plantillas.

Por último, la solución de escritorio Denim obtiene 11 puntos. Aunque iguala los mejores valores respecto a su operatividad (3), su baja puntación se justifica, principalmente, por su menor funcionalidad (6 puntos) y por la ausencia de plantillas $(0)$. 


\section{Herramientas en línea}

Una destaca sobre el resto: iPlo$t z$, aunque muy cerca de Mockflow. iPlotz obtiene la puntuación más alta del ranking, compartiéndola con las herramientas de escritorio. Presenta una funcionalidad mediaalta (11 puntos), pero obtiene esa posición en el ranking gracias al índice de operatividad más alto (5 puntos). Como ya hemos señalado, MockFlow ocupa el segundo lugar de ese ranking segregado separado solamente a un punto del primero. Presenta la misma tasa de funcionalidad que el anterior, pero una operatividad un poco más baja (3 puntos, el segundo valor más alto).

El siguiente grupo está formado por Pidoco y Lovely chart, con 12 puntos cada una. Estas soluciones obtienen sus puntaciones a partir de los parámetros funcionalidad $(7$, el tercer valor más bajo de todas las aplicaciones evaluadas), operatividad (3), soporte (2); pero fallan en el tema de las plantillas (2).

Al igual que ocurría en el ranking general, las soluciones en línea del final de la tabla son Mocking-
Bird y Lumzy, con 9 puntos cada una. Esa puntuación se basa sobre todo en los valores obtenidos en la funcionalidad (sólo 5 puntos). Aunque debe destacarse de estas últimas su extremada facilidad de uso.

Entendemos que diferentes colectivos de usuarios (entre los que no podemos dejar de considerar a los estudiantes de estas disciplinas) pueden beneficiarse de la información presentada aquí, ya que les permite, eventualmente, ayudarles en su toma de decisiones, incluso en el caso de las herramientas de coste cero.

Además, el grado de madurez y de sofistificación que alcanzan, incluyendo algunas opciones de coste cero en sus versiones basic, indican que es no solamente conveniente, sino altamente factible que los diseñadores de sitios y los arquitectos de la información se acostumbren a incorporar esta clase de programas e su cartera de recursos.

Agradecimiento: Este trabajo ha sido financiado parcialmente por el proyecto de investigación
CSO2009-13713-C05-04 del Ministerio de Ciencia e Innovación.

\section{Bibliografía citada}

Brown, Dan. Communicating design: developing web site documentation and planning, 2007. Berkeley: New Riders.

Hassan, Yusef; Martín-Fernández, Francisco J.; Iazza, Ghzala. "Diseño web centrado en el usuario: usabilidad y arquitectura de la información". Hipertext.net, 2004, n. 2.

http://www.hipertext.net

Khan, Saud. "Guidelines, tools and resources for web wireframes". W3avenue, feb. 2010. http://www.w3avenue.com/2010/02/03/guidelinestools-and-resources-for-web-wireframing/

Pedraza-Jiménez, Rafael. "Especificación de requisitos de un sitio web" [Informe]. Slideshare. net, oct. 2009.

http://www.slideshare.net/rpedraza/especifica cin-de-requisitos-de-un-sitio-web

Pérez-Montoro, Mario. Arquitectura de la información en entornos web, 2010. Gijón: Trea.

"Website wireframes". Wikipedia.

http://en.wikipedia.org/wiki/Website_wireframe

Mario Pérez-Montoro, Facultat de Biblioteconomia i Documentació, Universitat de Barcelona. perez-montoro@ub.edu

Lluís Codina, Departament de Comunicació, Universitat Pompeu Fabra. lluis.codina@upf.edu

\section{Spanish Meeting Point}

La revista El profesional de la información organiza una nueva edición del Spanish Meeting Point, dentro de la Online Information Conference, en Londres - la más importante del mundo en materia de contenidos y bases de datos científicas y profesionales, así como en software de gestión de contenidos.

\section{Online Information Conference, Londres 30 noviembre - 2 diciembre 2010}

http://www.online-information.co.uk

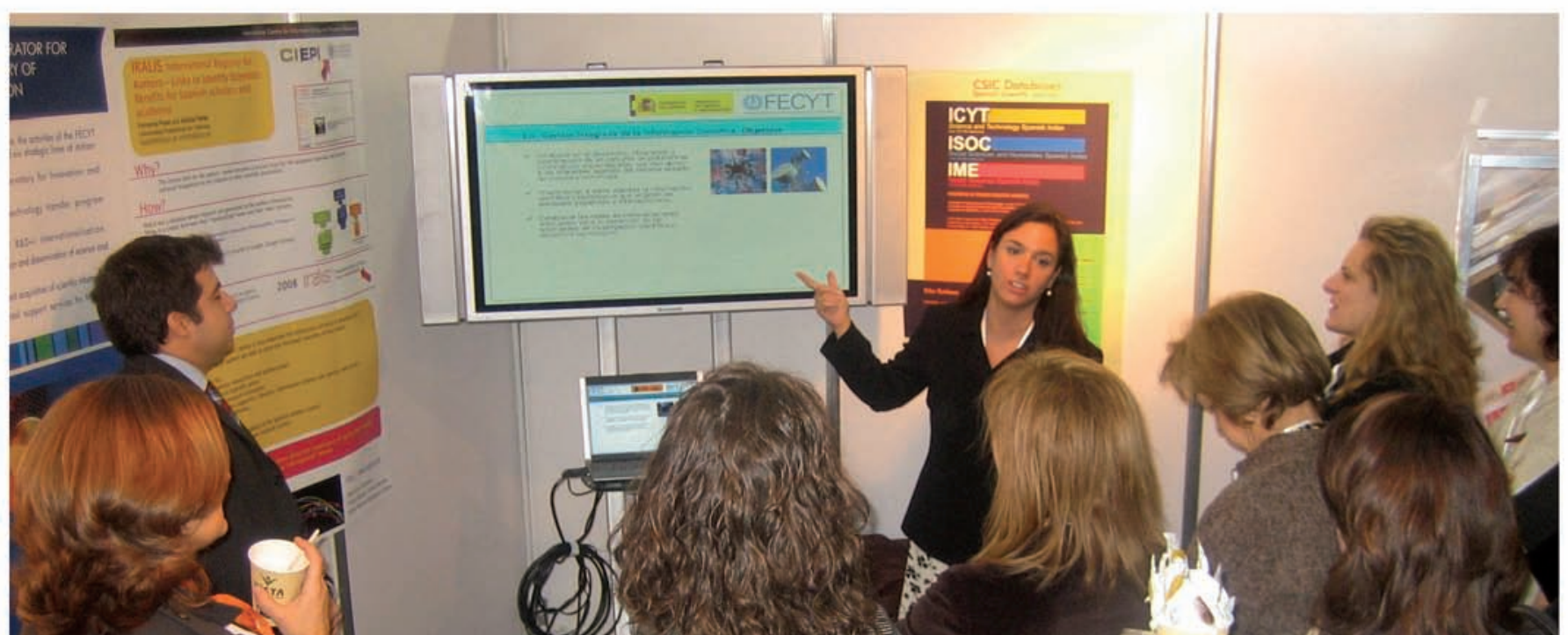

